\title{
Reconstruction of distal humerus with vascularized fibula after ewing's sarcoma resection - case report
}

\begin{abstract}
Ewing's sarcoma is one of the most common primary bone tumors of childhood, the tumor is almost always metaphyseal or diaphyseal, within long bones. The surgical treatment options for Ewing's sarcoma on the humerus range from amputation, non-biological reconstruction - like endoprosthesis, to biological reconstruction - like vascular proximal fibula autograft.

Reconstruction after resection of humeral sarcomas in the immature skeleton poses specific challenges to the surgeon. We present a case report of a pediatric patient with a diagnosis of Ewing's sarcoma in humeral shaft, submitted to oncologic resection of distal humerus and reconstruction of the bone defect with autologous vascularized fibular graft.
\end{abstract}

Keywords: Ewing's sarcoma, Vascularized fibula Bone defect
Volume 10 Issue 2 - 2018

\author{
Bernardo Vaz Peres Alves, ${ }^{4}$ Luis José Moura \\ Alimena, ${ }^{2}$ Alexandre David,' Gabriel Severo \\ da Silva, ${ }^{4}$ Cristian Stein, ${ }^{3}$ Daniel Dias de \\ Souza ${ }^{5}$ \\ 'Professor, Department of Orthopedics and Traumatology, \\ UFCSPA, Brazil \\ ${ }^{2}$ Assistant surgeon, Department of Orthopedic Oncology \\ Group, UFCSPA, Brazil \\ ${ }^{3}$ Assistant surgeon, Department Hand and Microsurgery, \\ UFCSPA, Brazil \\ ${ }^{4}$ Resident, Department of Orthopedics and Traumatology, \\ UFCSPA, Brazil \\ ${ }^{5}$ Medical School student, UFSM, Brazil
}

\begin{abstract}
Correspondence: Bernardo Vaz Peres Alves, Santa Casa de Misericordia de Porto Alegre, Department of Orthopedics and Traumatology, Rua Prof. Annes Dias 295 - Centro, CEP: 90020 090, Porto Alegre - RS, Brazil,

Email bernardovpalves@gmail.com
\end{abstract}

Received: August 31, 2017 | Published: January 25, 2018

\section{Introduction}

Ewing's sarcoma is the second most common primary malignant bone tumor in children. ${ }^{1,2}$ especially in white males. It is a highly aggressive tumor in the original primary site as well as in the metastasis spreading process, which demands fast diagnosis and appropriate treatment. $^{3}$ The clinical and radiological profile is quite similar to pediatric acute hematogenous osteomyelitis, which may hinder early diagnosis.$^{4}$ Therefore, diagnosis often demands bone biopsy. ${ }^{4}$ Fever and local inflammatory signals are commonly found in physical examination whereas anemia, leukocytosis, increased inflammatory markers, and lactate dehydrogenase (LDH) are commonly found in exams - the latter is also used as a chemotherapy response marker and tumor prognosis. ${ }^{5}$

Radiological findings show the tumor is most commonly found in the metaphyseal and diaphyseal regions of long bones - permeable osteolytic lesions and sometimes onion skin periosteal reaction. ${ }^{1,4}$ Other complementary exams such as bone scintigraphy, CT scans, and MRI are equally important when evaluating the extent of the sarcoma as well as the systemic repercussion. The most common treatment protocol is based on neoadjuvant chemotherapy, associated to surgery with wide tumor free margin and adjuvant chemotherapy. ${ }^{3,6,7}$ Radiotherapy is particularly suitable for tumors located in areas of difficult resection.

\section{Case report}

An 11-year-old male looked for medical assistance due to pain and swelling in his right arm, which had started three months earlier. Patient showed no other signs or symptoms. Swelling and warmth in the middle and distal thirds of the patient's arm were found in physical examination. All laboratory tests fell within normal values, i.e., within reference range values, except for the LDH level, which was $280 \mathrm{U} / \mathrm{L}$ (normal LDH levels range from $100 \mathrm{U} / \mathrm{L}$ to $190 \mathrm{U} / \mathrm{L}$ ). Radiological findings (Figures $1 \& 2$ ) showed permeable lytic lesions and "onion skin" periosteal reaction in the right humeral shaft. Thus, needle bone biopsy was indicated. Biopsy confirmed the presumptive diagnosis of Ewing's sarcoma. After staging, which excluded the dissemination of the disease, the treatment protocol was started, which was neoadjuvant chemotherapy, associated to surgery and adjuvant chemotherapy. The oncologic resection procedure was performed 5 (five) months after the diagnosis - arthrotomy of the right elbow and humerus osteotomy at $22 \mathrm{~cm}$ from the proximal extremity. In order to cover the bone defect, we opted for reconstruction with ipsilateral vascularized fibular autograft - a segment of $23 \mathrm{~cm}$ of the right fibula with a vascular pedicle to the fibular artery has been resected. The medical team then performed a termino-lateral anastomosis to the brachial artery and termino-terminal anastomosis to the basilic vein (Figure 3). Clamping of the fibular graft under pressure in the humeral medullary cavity was the method of choice for graft fixation in the proximal end, and distally, the fixation was obtained with capsulorrhaphy of the head of the fibula to the elbow joint (Figure 4). The choice not to use a plate for proximal fixation was made in the intraoperative period due to the satisfactory stability obtained with the clamping of the graft in the humerus. Patient presented good postoperative evolution, with a satisfactory range of mobility in the limb which underwent surgery, and high patient-satisfaction ratings (Figure 5). Two fractures of the grafted bone segment (the first occurred 13 months after the surgery and the other one 32 months after the surgery) were the postoperative complications reported. Both of them were satisfactorily handled with conservative treatment, i.e., full cast.

\section{Disscussion}

Bone reconstruction after oncologic resection of the sarcoma is challenging, especially when sparing the limbs is pivotal due to the bone defect which has been generated. Existing options for 
limb salvage are allografts, autologous grafts, and endoprosthesis. Autologous grafts with vascularized fibula is a biological choice to repair large bone defects. ${ }^{6,7}$ or pathological fractures in irradiated bones. ${ }^{8}$ Another autograft option is the clavicle bone flap, which is more indicated for bone defects in the ipsilateral proximal humerus. ${ }^{9}$ When compared to allografts, vascularized fibula shows fewer cases of pseudarthrosis, faster bone healing, and as a result, patients are able to resume everyday life activities in a shorter period of time. ${ }^{10}$ Presumably, such results occur due to mechanical stability which provides biological stimulus for consolidation. In some cases, it is possible to associate allografts and vascularized fibula, which is commonly used in cases of pseudarthrosis, fracture or infection in the reconstruction with allografts. ${ }^{11}$ We avoid the use of endoprosthesis in pediatric patients, except for cases in which prognosis is poor the presence of metastasis in the diagnosis or poor response to neoadjuvant chemotherapy; besides the incidence of revisions was higher for patients with prosthetic reconstruction. ${ }^{12}$ The expected instability in the elbow neo articulation has not occurred due to the great power of adaptation children have.
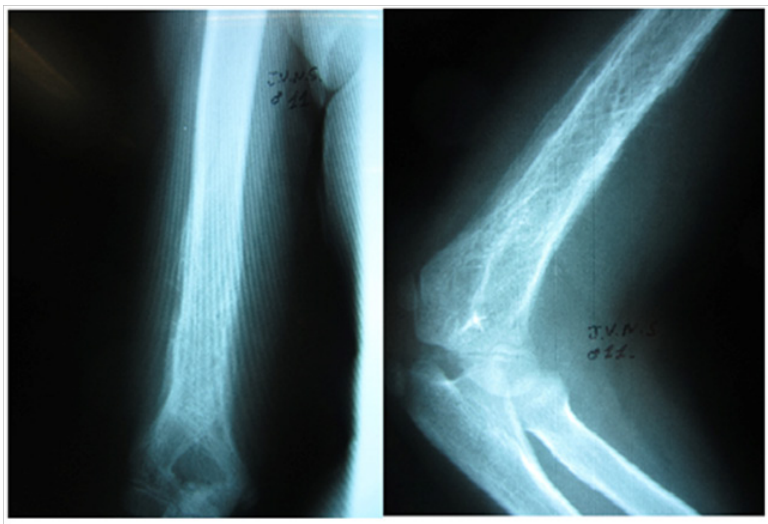

Figure I Anteroposterior X-ray view of the right humerus and lateral view of the right elbow. Permeable lytic lesion and "onion skin" periosteal reaction in distal humerus can be seen.

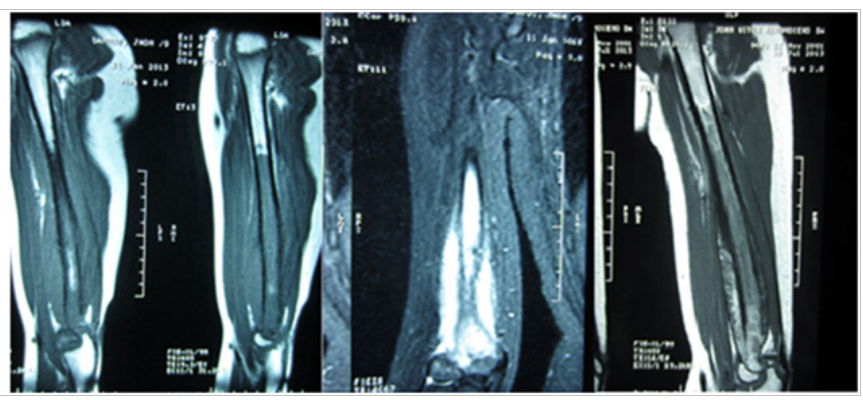

Figure 2 MRI of the right humerus is useful to better evaluate the extent of the tumor and plan the surgical procedure. The medullar limit of the tumor is shown in the sagittal sequence on the left. The image to the right shows better definition of tumoral limits due to satisfactory response to chemotherapy.

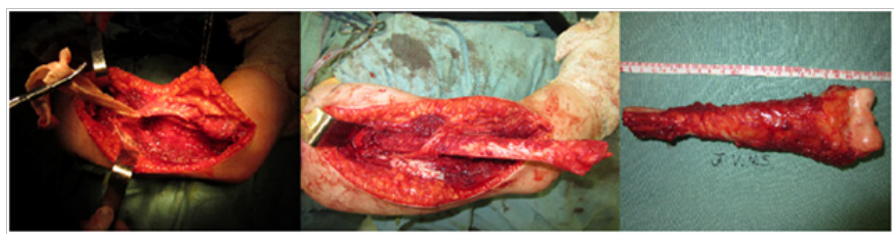

Figure 3 Bone defect after tumor resection on the left and reconstruction with vascularized fibula in the middle. The picture on the right shows the resected distal humerus.

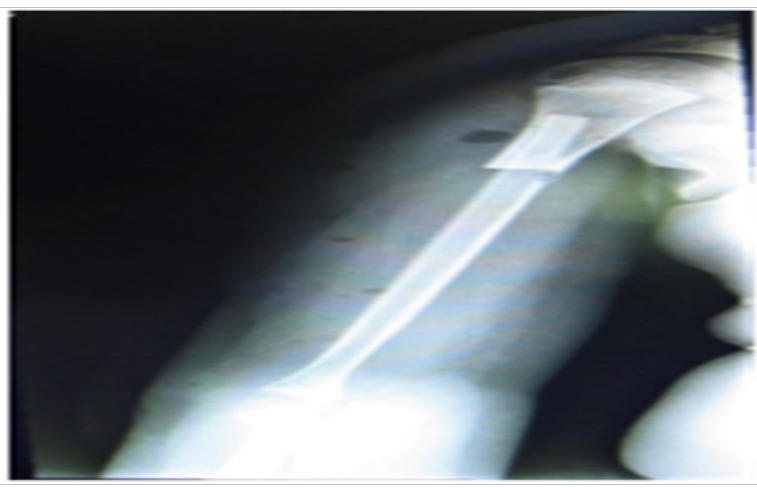

Figure 4 Anteroposterior view of the arm after the surgery. We can see the clamping of the fibula in the proximal medullary cavity of the humerus.

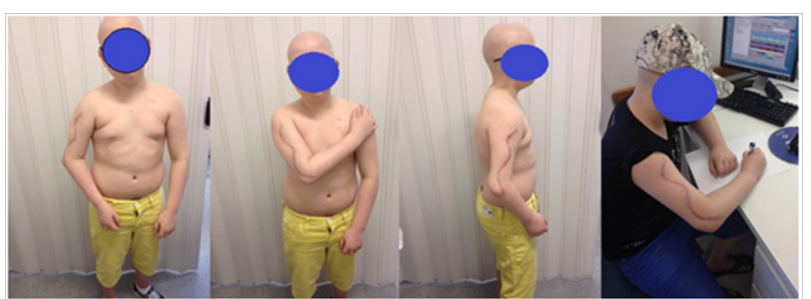

Figure 5 Photographs of the limb 5 (five) months after surgery (left) and 8 (eight) months after the surgery (right). The patient has satisfactory function with ative movement of the elbow.

\section{Conclusion}

Regardless of the complications which may occur in up to half of the cases. ${ }^{13}$ bone reconstruction with vascularized fibula is an option for limb salvage, which is a lasting solution, especially for pediatric patients. Limb-salvage surgery must not worsen the original primary site or distant (metastasis) outcomes of the disease when compared to limb amputation. ${ }^{14}$ and also shows good patient-satisfaction ratings as most patients are able to resume everyday life activities.

\section{Conflicts of Interest}

None.

\section{Acknowledgements}

None.

\section{References}

1. Biswas B., Bakhshi S Management of Ewing sarcoma family of tumors: Current scenario and unmet need. World J Orthop. 2016;7(9):527-538.

2. Esmaili HA, Niknejad MT, Mohajeri S Ewing's sarcoma of proximal humeral epiphysis. Arch Iran Med. 2015;18(2):133-134.

3. Manfrini M, Tiwari A, Ham J et al. Evolution of surgical treatment for sarcomas of proximal humerus in children: retrospective review at a single institute over 30 years. J Pediatr Orthop. 2011;31(1):56-64.

4. McCarville MB, Chen JY, Coleman JLet al. Distinguishing Osteomyelitis From Ewing Sarcoma on Radiography and MRI. AJR Am J Roentgenol. 2015;205(3):640-650, quiz 651.

5. Li S, Yang Q, Wang $\mathrm{H}$ et al. Prognostic significance of serum lactate dehydrogenase levels in Ewing's sarcoma: A meta-analysis. Mol Clin Oncol. 2016;5(6):832-838.

6. Onoda S, Sakuraba M, Asano T et al. Use of vascularized free fibular head grafts for upper limb oncologic reconstruction. Plast Reconstr Surg. 2011;127(3):1244-1253. 
7. Belt PJ, Dickinson IC, Theile DR Vascularised free fibular flap in bone resection and reconstruction. Br J Plast Surg. 2005;58(4):425-430.

8. Friedrich JB, Moran SL, Bishop AT et al. Vascularized fibula flap onlay for salvage of pathologic fracture of the long bones. Plast Reconstr Surg 2008;121(6):2001-2009.

9. Calvert GT, Wright J, Agarwal J et al. Is claviculo pro humeri of value for limb salvage of pediatric proximal humerus sarcomas? Clin Orthop Relat Res. 2015;473(3):877-882.

10. Friedrich JB, Moran SL, Bishop AT et al. Free vascularized fibula grafts for salvage of failed oncologic long bone reconstruction and pathologic fractures. Microsurgery. 2009;29(5):385-392.

11. Houdek MT, Wagner ER, Stans AA et al. What Is the Outcome of Allograft and Intramedullary Free Fibula (Capanna Technique) in Pediatric and Adolescent Patients With Bone Tumors? Clin Orthop Relat Res. 2016;474(3):660-668.
12. Liu T, Zhang Q, Guo X et al. Treatment and outcome of malignant bone tumors of the proximal humerus: biological versus endoprosthetic reconstruction. BMC Musculoskelet Disord.2014;15:69.

13. Rose PS, Shin AY, Bishop AT, Moran SL, Sim FH (2005) Vascularized free fibula transfer for oncologic reconstruction of the humerus. Clin Orthop Relat Res. 2014;438:80-84.

14. Eward WC, Kontogeorgakos V, Levin LS et al. Free vascularized fibular graft reconstruction of large skeletal defects after tumor resection. Clin Orthop Relat Res. 2010;468(2):590-598. 